\title{
Protective role of fingolimod (FTY720) in rats subjected to subarachnoid hemorrhage
}

\author{
Hao-Liang Xu' ${ }^{1}$, Dale A Pelligrino ${ }^{1}$, Chanannait Paisansathan² and Fernando D Testai ${ }^{3^{*}}$
}

\begin{abstract}
Background: Subarachnoid hemorrhage $(\mathrm{SAH})$ is a neurological emergency with limited pharmacological treatment options. Inflammation is increasingly recognized as a key pathogenic contributor to brain injury in this condition. In the present study, we examined the neuroprotective effects of the immunomodulatory agent, fingolimod, in rats subjected to SAH.

Methods: We utilized an endovascular rat perforation model of SAH. Animals were divided into four groups: (1) sham-vehicle; (2) sham-fingolimod; (3) SAH-vehicle; and (4) SAH-fingolimod. Rats received either vehicle solution or fingolimod $(0.5 \mathrm{mg} / \mathrm{kg})$ intraperitoneally 3 hours after sham surgery or SAH. A closed cranial window and intravital microscope system was used at 48 hours to assess neuroinflammation, which was represented by rhodamine-6Glabeled leukocyte trafficking in pial venules, and pial arteriolar dilating responses to a variety of vasodilators, including hypercapnia, and topically-applied acetylcholine, adenosine, and S-nitroso-N-acetyl penicillamine. In addition, motor-sensory function was evaluated.

Results: Compared to sham-vehicle rats, SAH-vehicle animals displayed a four-times greater increase in pial venular intraluminal leukocyte adhesion. Treatment with fingolimod largely reduced the intravascular leukocyte adhesion. Vehicle-treated SAH animals displayed a significant decrease in pial arteriolar responses to all the vasodilators tested and vascular reactivity was preserved, to a significant degree, in the presence of fingolimod. In addition, neurological scores obtained at 48 hours post-SAH indicated significant neurological deficits in the vehicle-treated group (versus sham-vehicle surgical control). Those deficiencies were partially reduced by fingolimod $(P<0.0001$ compared to the vehicle-treated SAH group).
\end{abstract}

Conclusions: Treatment of rats with fingolimod was associated with a marked limitation in the intravascular adhesion of leukocytes to pial venules, preserved pial arteriolar dilating function, and improved neurological outcome in rats subjected to SAH.

Keywords: Subarachnoid hemorrhage, Microvascular function, Fingolimod, Neuroinflammation

Subject codes: Cerebrovascular disease/stroke, Subarachnoid hemorrhage

\section{Introduction}

Subarachnoid hemorrhage (SAH) is associated with a disproportionately elevated morbidity and mortality. The overall case fatality is estimated in 40 to $50 \%$ with almost 10 to $15 \%$ of the patients dying before reaching medical attention $[1,2]$. Of the survivors, $46 \%$ may have longterm cognitive impairment and 30\% require lifelong supportive care $[3,4]$. This condition often affects young

\footnotetext{
*Correspondence: testai@uic.edu

${ }^{3}$ Department of Neurology and Rehabilitation of the University of Illinois College of Medicine, 912 S Wood Street, Rm 855 N NPI (MC 796), Chicago, IL 60612-7330, USA

Full list of author information is available at the end of the article
}

adults and may be responsible for almost a quarter of all the years lost because of stroke [5]. Despite intense research, progress toward the development of effective treatments for SAH has been disappointingly slow. Nimodipine remains the only pharmacological treatment for this condition. The beneficial effect of nimodipine, however, is limited and its use is often restricted by the occurrence of hypotension [6]. New and effective treatments for SAH are largely needed. Studies performed over the past decade have yielded a preponderance of evidence supporting a link between inflammation and stroke outcome [7-10]. Inflammation plays an important 
role in brain damage by contributing to neural injury, diminished vascular reactivity, microthrombosis, and enhanced blood brain barrier (BBB) permeability $[7,8,11,12]$. These observations support immunomodulation as a potential target for intervention following SAH. Fingolimod (FTY720) is an immunomodulatory agent has been approved by the Food and Drugs Administration (FDA) for the treatment of multiple sclerosis. Fingolimod is a sphingosine-1-phosphate (S1P) analog that crosses the $\mathrm{BBB}$ and regulates critical cellular processes including proliferation, apoptosis, endothelial barrier permeability, and inflammation [13,14]. Studies performed in association with cerebral ischemia and intraparenchymal hemorrhage have shown that FTY720 reduces stroke-related neuroinflammation, brain edema, and neuronal death and improves neurological outcome [15-17]. Thus, we hypothesized that fingolimod would be efficacious in the treatment of SAH. To that end, we investigated the effect of FTY720 in a rat model of SAH. In particular we addressed leukocyte adhesion to pial venules, microvascular (pial arteriolar) dilating reactivity, and neurological outcome.

\section{Methods}

\section{Drugs and SAH model}

FTY720 was supplied by Cayman Chemicals (Ann Arbor, MI, USA). Experimental protocols were approved by the institutional Animal Care and Use Committee of the University of Illinois at Chicago. Sprague-Dawley male rats $(250$ to $300 \mathrm{~g})$ were randomly assigned to the shamvehicle $(\mathrm{n}=10)$, sham-FTY720 $(\mathrm{n}=10)$, SAH-vehicle $(n=11)$, or SAH-FTY720 $(n=11)$ groups. We utilized the endovascular perforation model of SAH as previously described [18]. Briefly, animals were anesthetized with $2 \%$ isoflurane and mechanically ventilated. Physiological variables, including blood pressure, blood gases, and body temperature, were continuously monitored and kept within physiological ranges. Regional cerebral blood flow (rCBF) was monitored before, during, and after SAH by Laser Doppler flowmetry (LDF), which entailed attachment of a laser-Doppler probe to the skull over the right middle cerebral artery (MCA) territory. The right internal (ICA) and external carotid arteries (ECA) were isolated to their origins at the common carotid artery (CCA) bifurcation. The ECA was ligated and shaped into a short stump. The CCA was temporary clipped, and a hollow polytetrafluoroethylene (PTFE) tube was advanced rostrally into the ICA from the ECA stump until resistance was felt, and then a tungsten wire was partially advanced through PTFE tube to perforate the bifurcation of the anterior and middle cerebral arteries. Immediately after puncture, the PTFE tube and tungsten wire were retracted into the ECA stump, and the ICA was reperfused. The incision was then closed with nylon monofilament suture, and rats were extubated and returned to their cages. SAH was determined by a transient drop in cerebral blood flow of $>95 \%$ and confirmed by post-mortem examination which was performed at 48 hours post-surgery. Blood accumulation at the circle of Willis indicates a successful vessel perforation. Similar surgical procedure was performed in sham-vehicle and FTY720 groups except that the suture was removed once resistance was felt and no tungsten wire perforation was performed. To minimize factors that may influence bioavailability we used the intraperitoneal (IP) route. Animals were treated with vehicle or FTY720 $(0.5 \mathrm{mg} / \mathrm{kg})$ at 3 hours after surgery.

\section{Peripheral leukocyte count}

The effect of FTY720 on leukocytes was determined using peripheral blood samples. Animals were randomly assigned to sham-vehicle $(\mathrm{n}=3)$, sham-FTY720 $(\mathrm{n}=5)$, SAH-vehicle $(\mathrm{n}=5)$, and SAH-FTY720 $(\mathrm{n}=4)$ groups. Blood samples were collected before (baseline) and 48 hours after surgery. An additional non-surgical naïve control group was used to confirm the immonomodulatory effect of FTY720. A total of $0.5 \mathrm{ml}$ of blood was obtained from the subclavian vein, placed in a Microtainer tube coated with $K_{2}$-EDTA (BD Biosciences, San Jose, CA, USA), and analyzed using an automated hematology analyzer Advia 120 (Siemens, Dublin, Ireland).

\section{Cranial window installation}

Cranial windows were installed $48 \mathrm{~h}$ after surgery as previously described. Briefly, bilateral femoral arterial and venous catheters were placed with the rats under $2 \%$ isoflurane $/ 70 \% \mathrm{~N}_{2} \mathrm{O} / 30 \% \mathrm{O}_{2}$ anesthesia, paralyzed with curare $(1 \mathrm{mg} / \mathrm{kg}$ intravenously) and supported with mechanical ventilation. Femoral artery and vein were canulated for arterial blood pressure monitoring and arterial blood gas measurement. Body temperature was kept at $37^{\circ} \mathrm{C}$. The animal was positioned on a stereotactic frame and a $10 \mathrm{~mm}$ craniotomy was performed over the skull midline. The dura was removed carefully to keep the sagital sinus intact. An $11 \mathrm{~mm}$ in diameter and $1.5 \mathrm{~mm}$ in thickness borosilicate glass window equipped with 3 ports was glued to the skull. The time from dural excision to sealing of the cranial window was minimized (less than 5 minutes) to avoid complications from brain swelling and damage at the dural edge. The intracranial pressure (ICP) under the cranial window was monitored thorough the experiment using the ICP measurement port. Artificial CSF (aCSF) was suffused into the cranial window and the effusion rate was adjusted to maintain an ICP of 7 to $11 \mathrm{mmHg}$ in all groups. After completion of the surgery, the anesthesia with isoflurane was switched to fentanyl infusion for monitoring of rhodamine-6G (R6G) leukocyte labeling and the assessments of pial arteriolar responses. 


\section{Leukocyte behavior analysis}

Leukocyte behavior analyses were performed 48 hours after surgery by infusing R6G as previously described [19]. This is a semiquantitative method used for the in vivo assessment of leukocyte trafficking. Fluorescence area of R6G-labeled leukocytes adhered to the vessel wall (this includes both tightly-adhered and slow-rolling leukocytes) is used as a surrogate marker of neuroinflammation. Animals were anesthetized with $70 \% \mathrm{~N}_{2} \mathrm{O}$ / $30 \% \mathrm{O}_{2}$ - fentanyl $(25 \mu \mathrm{g} / \mathrm{kg} / \mathrm{h}$, intravenously). After cranial window placement, R6G (0.2 mg loading dose) was applied intravenously to sham-vehicle, sham-FTY720, SAH-vehicle, and SAH-FTY720 groups, followed by 0.2 $\mathrm{mg} / \mathrm{h}$ maintenance dose ( $\mathrm{n}=6$ per group). Pial venules ranging from 40 to $80 \mu \mathrm{m}$ in diameter were randomly selected. R6G-labeled stationary leukocytes were viewed and captured using a digital camera (CoolSnap ES, Photometrics, Tucson, AZ, USA) mounted on a fluorescence microscope (Nikon, Melville, NY, USA) equipped with an epi-illumination dark field system. Images were obtained 60 minutes after initiating R6G infusion and analyzed using MetaMorph software (Molecular Devices, Downingtown, PA, USA). R6G dosage, intravenous suffusion time, and laser light source were applied in identical manner to each animal, minimizing the variations in leukocyte labeling. Using this technique, only adherent leukocytes can be detected [20]. Leukocyte adhesion was reported as the \% of adherent leukocytes occupying the viewed venular area in the microscopic field.

\section{Arteriolar reactivity}

Vascular reactivity was determined 48 hours after SAH by measuring pial arteriolar diameter changes in response to different vasodilating stimuli, including hypercapnia, acetylcholine (Ach, $10 \mu \mathrm{M}$ and $100 \mu \mathrm{M}$ ), adenosine (ADO, $10 \mu \mathrm{M}$ and $100 \mu \mathrm{M}$ ), and S-nitroso-N-acetyl penicillamine (SNAP, $0.1 \mu \mathrm{M}$ and $1 \mu \mathrm{M}$ ) as previously described [19,21]. Briefly, animals were randomly assigned to the shamvehicle, sham-FTY720, SAH-vehicle, and SAH-FTY720 groups ( $\mathrm{n}=5$ to 6 per group). Pial arteriolar diameters were measured through a cranial window after 40 minutes of suffusion of aCSF (baseline) and again after 3 minutes of hypercapnia $\left(\mathrm{PaCO}_{2}\right.$ at approximately $\left.70 \mathrm{mmHg}\right) \cdot \mathrm{CO}_{2}$ reactivity was calculated as the percentage of diameter increase per $\mathrm{mmHg}$ of $\mathrm{PaCO}_{2}$ change. Fifteen minutes after return to baseline, Ach, ADO, and SNAP were sequentially suffused in the space under the cranial window at the concentrations indicated. Diameter values from three arteriolar segments were obtained and averaged.

\section{Neurological assessments}

These were done at 48 hours post-SAH by a blinded observer prior the placement of the cranial window. We utilized a 21-point scale as previously described [20].
Briefly, animals were randomly assigned to the shamvehicle, sham-FTY720, SAH-vehicle, and SAH-FTY720 groups ( $\mathrm{n}=10$ to 11 per group). The ratings were taken from a sum of scores from 7 categories: (i) spontaneous activity, (normal $=3$, akinesia $=0$ ); (ii) side stroking (bilateral brisk response $=3$, no response $=0$ ); (iii) vibrissae touch (bilateral brisk response $=3$, no response $=0$ ); (iv) limb symmetry (forelimb and hindlimb extended $=3$, contralateral forelimb and hindlimb completely flexed $=0$ ); (v) lateral turning (bilateral turning $\geq 45^{\circ}=3$, no turning $=0$ ); (vi) forelimb walking (briskly walks forward in symmetry $=3$, cannot move on forelimbs $=0$ ); and (vii) slope climbing (climbs to the top of slope with prompting and strong grip $=3$, weak grips and falls off $=0$ ).

\section{Statistics}

Group sizes were determined based on power analysis. In our calculations we assumed a $30-50 \%$ change in the mean, 20\% standard deviation (SD), alpha of 0.05 , and power of 0.8. Based on power analysis the minimum group sizes were 5. Peripheral leukocyte counts were expressed as means \pm SD and analyzed using an unpaired Student $t$-test. Venular leukocyte adhesion, pial arteriolar reactivity, and neurological outcome were expressed as means \pm standard error of the mean (SEM). The effect of FTY720 on on these variables was analyzed using one-way analysis of variance (ANOVA). Significance was defined as $P<0.05$.

\section{Results}

\section{Physiological variables for the SAH animal model}

The overall mortality after SAH was approximately $30 \%$. Of the animals that survived the SAH surgery, one animal died in the SAH-vehicle group and one in the SAHFTY720 group. Blood pressure, body temperature, and blood gas parameters were similar in the four groups. CBF decreased abruptly by 85 to $90 \%$ after SAH. The nadir occurred within the first minute of $\mathrm{SAH}$ and this was followed by a gradual recovery reaching a plateau at approximately $60 \%$ of baseline values within 20 minutes. The cerebral hemodynamic responses were comparable in both the vehicle- and FTY720-groups. SAH was also confirmed by post -mortem visual inspection (Figure 1).

\section{Effect of FTY720 on circulating blood cell counts}

The treatment with a single dose of $0.5 \mathrm{mg} / \mathrm{kg}$ FTY720 in non-surgical animals resulted in a steep decrease in circulating white blood cells at both 24 hours and 48 hours (Figure 2A). This decrease was largely driven by lymphocyte depletion, which decreased to $15 \%$ and $12 \%$ from the basal levels measured at 24 hours and 48 hours post-treatment, respectively $(P<0.001)$. Blood samples in sham and SAH-exposed animals were obtained at baseline and 48 hours after surgery. In comparison to 


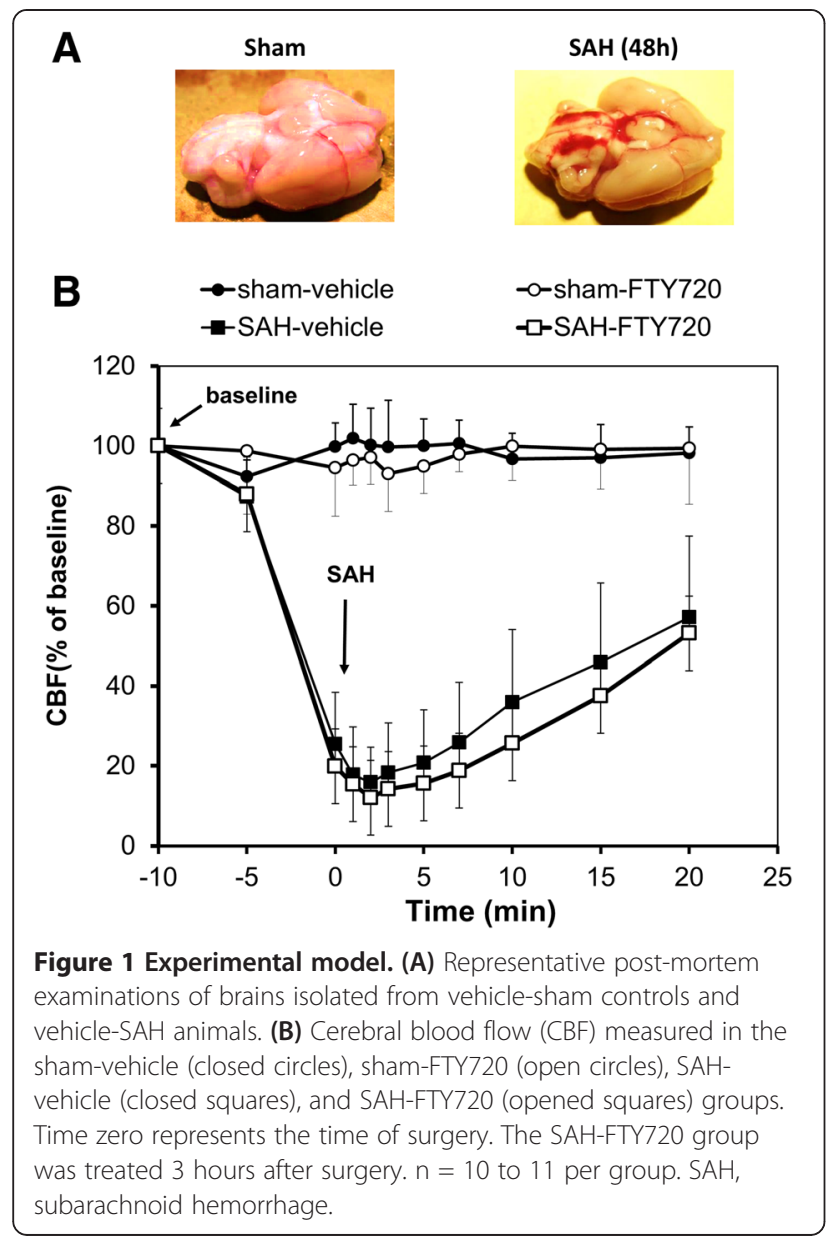

vehicle-SAH animals, a single dose of $0.5 \mathrm{mg} / \mathrm{kg}$ FTY720 3 hours after sham- or SAH-surgery was associated with a statistically significant decrease in total leukocytes and lymphocytes in peripheral blood (Figure $2 \mathrm{~B}$ and $\mathrm{C}$ ).

\section{Leukocyte adhesion}

Circulating leukocytes were tagged with R6G and videorecorded at 48 hours post-SAH. Representative frames were captured from video-recordings (Figure 3). Leukocyteadhesion in the sham-vehicle and sham-FTY720 groups were $2.9 \pm 0.2 \%$ and $1.3 \pm 0.5 \%$, respectively. Vehicle-SAH animals displayed almost an approximately 5 -fold increase in vascular leukocyte adhesion to pial venules compared to controls $(14.4 \pm 1.0 \% ; P<0.0001)$. Treatment with FTY720, on the other hand, was associated with a decreased SAH-induced leukocyte adhesion by approximately $40 \%(8.8 \pm 2.2 \%)$.

\section{Arteriolar reactivity}

Cerebral microvascular dilating function was determined by assessing arteriolar reactivity to established vasodilators, including hypercapnia $\left(\mathrm{PaCO}_{2}\right.$ at approximately 70 $\mathrm{mmHg})$, Ach $(10 \mu \mathrm{M}$ and $100 \mu \mathrm{M})$, ADO $(10 \mu \mathrm{M}$ and

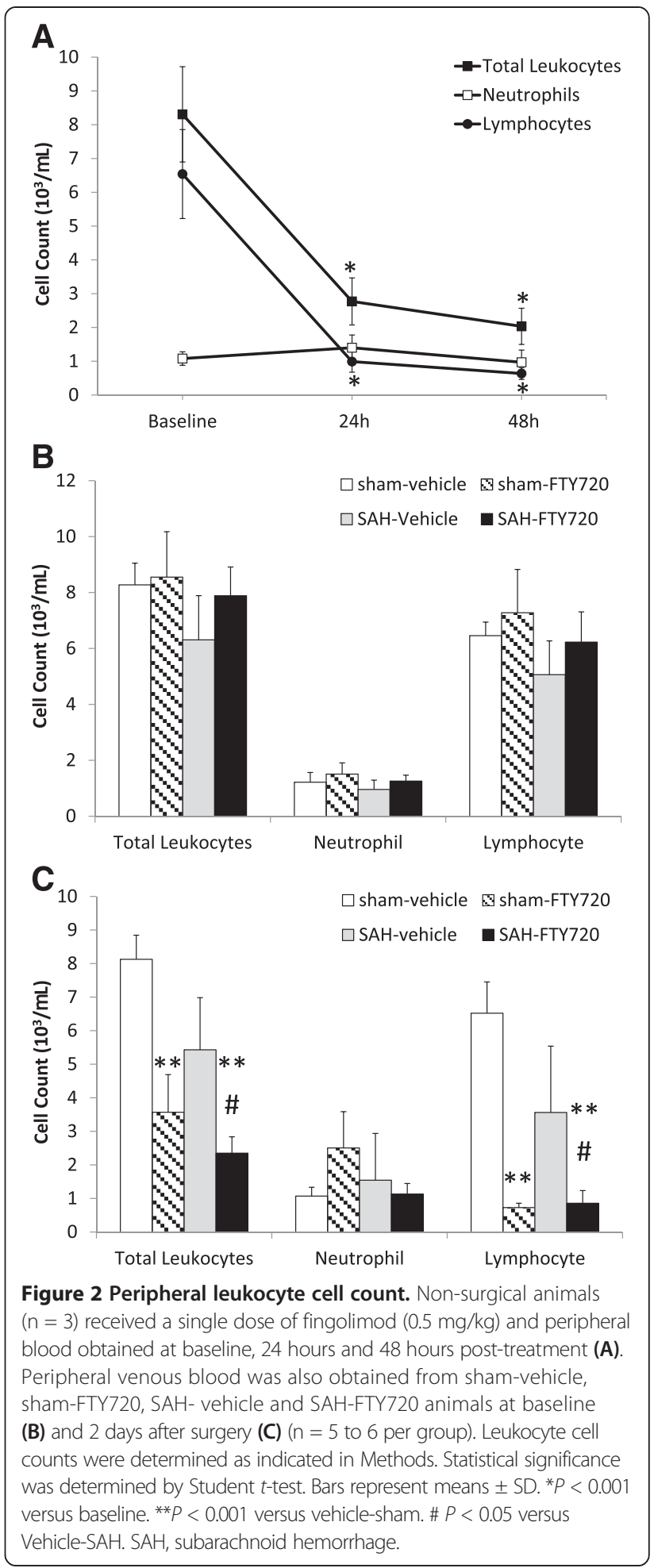

$100 \mu \mathrm{M})$, and SNAP $(0.1 \mu \mathrm{M}$ and $1 \mu \mathrm{M})$. Time-dependent alterations in arteriolar reactivity occur in SAH [22]. These changes reach their maximum 48 hours and gradually recover in the following 7 days after the hemorrhage. Hence, arteriolar reactivity was determined at 48 hours post-SAH. 


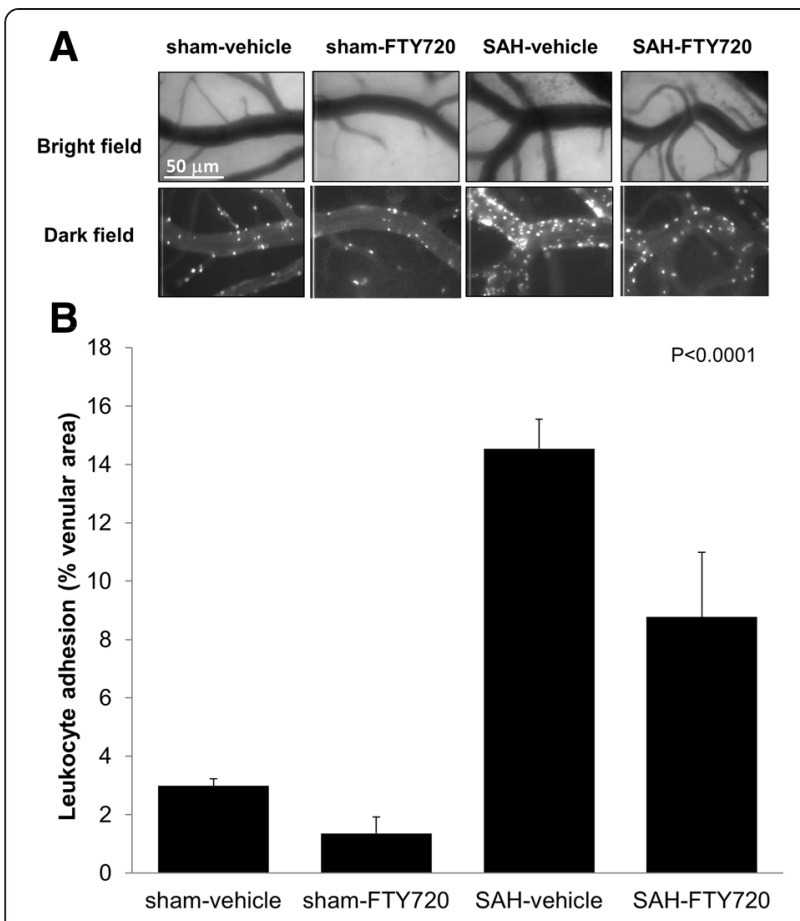

Figure 3 Leukocyte vascular adhesion. Animals were treated with vehicle or FTY720 (0.5 mg/kg) 3 hours post-surgery and leukocyte adhesion was determined at 48 hours. (A) Representative pictures of the vessel anatomy (bright field) and the trafficking of leukocytes (dark field) 1 hour after leukocyte labeling with R6G in sham-vehicle, shamFTY720, SAH-vehicle, and SAH-FTY720-treated animals. (B) Quantification of leukocyte adhesion, expressed as the percentage of the vessel area occupied by adherent leukocytes measured 1 hour after leukocyte labeling. Bars represent means \pm SEM. $n=6$ each group. Significance was determined using ANOVA. SAH, subarachnoid hemorrhage.

The arteriolar responses in the sham-vehicle and shamFTY720 were comparable. SAH-vehicle animals showed a profound attenuation of pial arteriolar responses to all the vasodilators tested. In comparison, SAH-FTY720 animals displayed a statistically significant preservation of arteriolar dilating function to all the agents investigated (Figure 4). The improvement was partial for hypercapnia and virtually complete for Ach, ADO, and SNAP.

\section{Neurological outcome}

Functional outcome was determined at 48 hours postSAH (Figure 5). The neurological score in sham-vehicle and sham-FTY720 groups were not statistically different $(20.5 \pm 0.4$ versus $20.6 \pm 0.4)$. In comparison, the score was reduced to $11.4 \pm 2.4$ in the $\mathrm{SAH}$-vehicle group. The treatment with FTY720 at 3 hours after SAH was associated with an improvement of $35 \%$ in neurological outcome $(15.5 \pm 1.8 ; P<0.0001)$.

\section{Discussion}

In this study we demonstrated that the use of fingolimod was associated with a restricted leukocyte adhesion to pial vessels, preserved arteriolar vasodilation responses, and improved neurological function after SAH.

FTY720 has been used with success in models of cerebral ischemia and parenchymal hemorrhage [15-17]. This, however, is the first study addressing the protective effect of this drug in animals subjected to SAH. The dose of FTY720 is in agreement with studies done in non-SAH models $[15,17,23,24]$. SAH is characterized by acute headache resulting in patients seeking early medical attention. Therefore, the 3 hours post-SAH treatment time point constitutes a therapeutic window similar to that encountered in clinical practice.

FTY720 is an agonist of S1P receptors type 1, 3, 4 and 5 which are ubiquitously distributed in cells of different lineages. Fingolimod induces the internalization and subsequent ubiquitination and proteosomal degradation of S1P-receptor type 1 on lymphocytes, which are retained in lymph organs [25]. In our study, FTY720 decreased circulating lymphocytes with virtually no effect on neutrophils, an observation that is consistent with the known mechanism of action of this drug [25,26]. FTY720 has a high volume of distribution and its halflife is of approximately 9 to 10 days [14]. Thus, it was not unexpected that a single dose of this drug led to an immunosuppressive state that persisted for at least 48 hours. In addition, the treatment with FTY720 restricted the adhesion of leukocytes to pial vessels and improved neurological outcome after SAH. The role of leukocytes in $\mathrm{SAH}$ is not well known. Immediately after $\mathrm{SAH}$, there is a rapid increase in the ICP leading to transient global hypoperfusion with subsequent restoration of the cerebral blood flow [27]. In this context, SAH has some similarities with transient cerebral ischemia. Studies performed in ischemic models illustrate that tissue hypoxia upregulates the expression of adhesion molecules that facilitate the interaction of circulating leukocytes with vascular elements. These release vasoactive and proinflammatory mediators that contribute to brain damage $[10,28,29]$. In $\mathrm{SAH}$, the initial insult induces an inflammatory state characterized by early recruitment of leukocytes and increased production of cytokines and chemokines in both plasma and CSF $[9,12,30]$. This immune response correlates with SAH severity and has been linked to neural cell injury, BBB dysfunction and delayed cerebral ischemia [31-33]. Accordingly, immunomodulation has emerged as a potential treatment strategy that could ameliorate brain injury and improve outcome after SAH. Our previous findings indicate that blocking leukocyte adhesion and transmigration into the brain parenchyma via LJP-1586, a selective vascular adhesion protein-1 (VAP-1) inhibitor, improves neurological outcome in SAH [34]. LJP-1586 is nonspecific and inhibits the adhesion of neutrophils, monocytes and lymphocytes. In comparison, FTY720 has a selective effect on lymphocytes. Studies performed in 


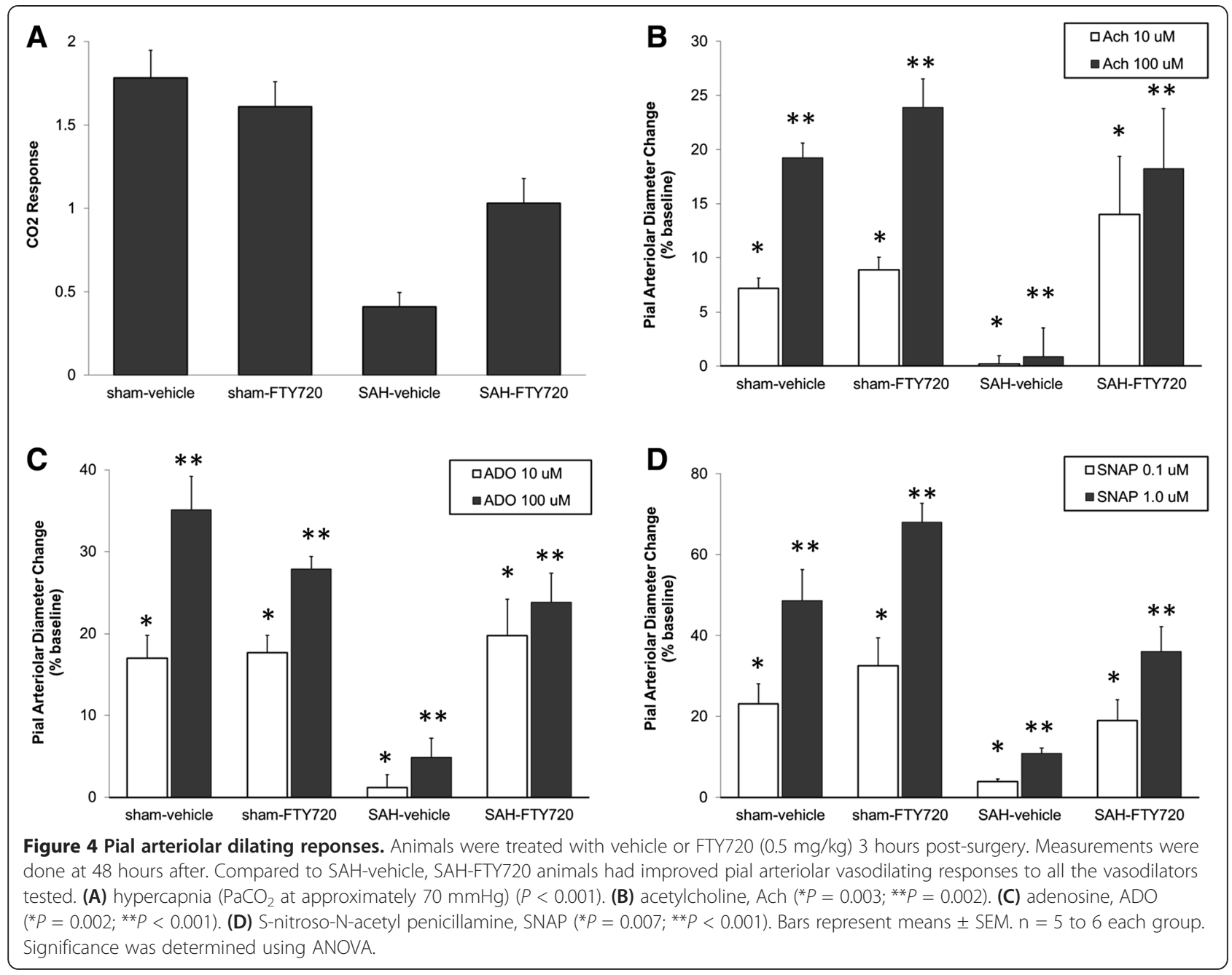

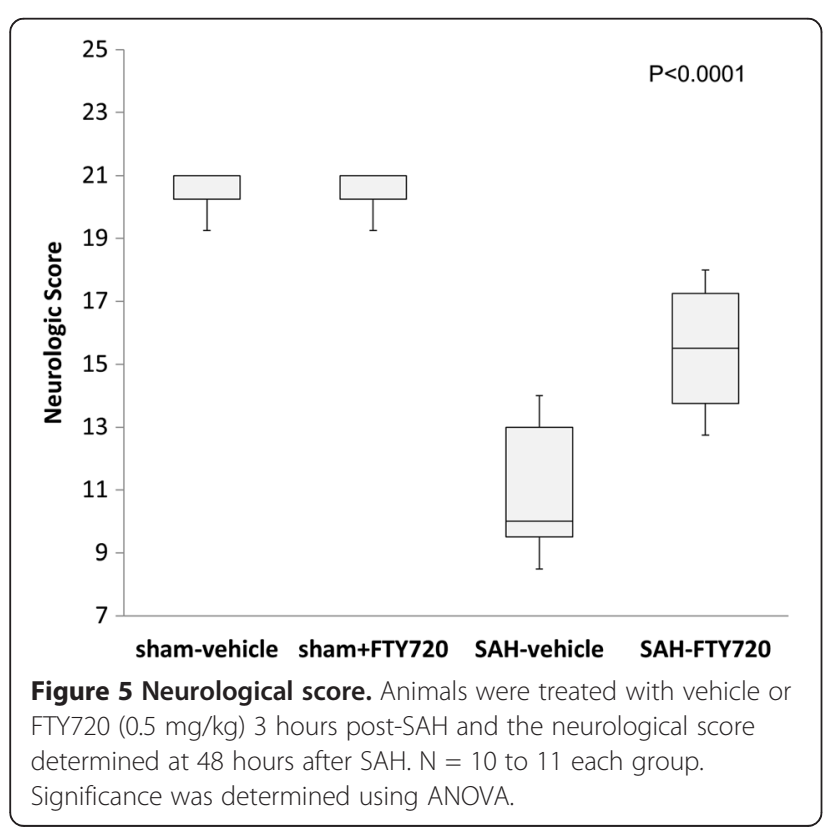

ischemic models confirm the contribution of both innate and adaptive immunity to stroke outcome. Understanding the role of lymphocytes in cerebral ischemia is confounded by the existence of specific subpopulations that are either protective, deleterious, or both [29]. Despite the limited information regarding the specific contribution of lymphocytes to SAH outcome, the selective effect of FTY720 supports the key role of this cell type on SAHassociated brain injury. Animal models, however, do not necessarily recapitulate the complex pathogenic mechanisms that take place in humans. From the immunological standpoint, the predominant circulating leukocytes in humans are neutrophils (50 to $70 \%$ ) and in rodents lymphocytes (60 to 80\%) [35]. In addition, immune-related molecules are differentially expressed in different species [35]. Therefore, the results obtained in experimental models may not necessarily apply to humans. Both similarities and differences have been described in the immune responses triggered by SAH in rodents and humans [31]. Significantly, it was observed that methylprednisolone, a drug that preferentially targets lymphocytes, is beneficial 
in rodents and patients with SAH [36-38]. The studies were too small to draw solid conclusions but they support the active role of lymphocytes in $\mathrm{SAH}$-associated brain injury.

In our study we also observed that the use of FTY720 preserved microvascular dilating function which was tested using different vasodilating agents. Hypercapnia and Ach induce cerebral arteriolar dilation via nitric oxide (NO) synthase/cyclooxygenase and endotheliumdependent mechanisms, respectively. In comparison, ADO tests G-coupled receptor-dependent vascular smooth muscle responses and SNAP interrogates vascular smooth muscle NO reactivity. Time-dependent alterations in the response of small arteries to vasodilators occur within minutes of the SAH and peak at 48 hours [22]. The upstream processes governing vascular tone on SAH are not completely understood. An association between inflammation and abnormal vasomotor responses in $\mathrm{SAH}$ has been established in large conduit arteries [39,40]. Lymphocytes, in particular, have been associated with the emergence of late vasospasm [41]. Pharmacological blockage of VAP-1 and neutrophil inhibition, in addition, ameliorate microvascular dysfunction in animal models suggesting that immunological factors regulate vasoreactivity in both small and large intracranial vessels [39]. Interestingly, there is evidence supporting the notion that microvascular dysfunction is a major contributor to microthrombi formation, tissue hypoxia, and outcome after SAH [42-44]. The improvement in neurological outcome, in parallel with decreased neuroinflammation and preserved arteriolar responses observed in association with FTY720, is in agreement with this paradigm. It should be pointed out, however, that this drug has a direct stabilizing effect on the endothelium and has been shown to enhance the endothelial synthesis of NO in non-neural tissues [14,45]. In our study the arteriolar responses in the sham-vehicle and sham-FTY720 were comparable $(P>0.05)$ indicating that, under the conditions used in this study, FTY720 does not have a direct vasodilating effect. FTY720 has a direct epigenetic effect via histone deacetylases regulation and also targets different components of the neurovascular unit. In particular, it inhibits astrogliosis, increases neuronal survival in cerebral ischemia, and enhances endothelial-barrier function [25,46-48]. Therefore, the preserved arteriolar function and neurological improvement observed in our study cannot be conclusively ascribed to the inhibition of lymphocytes.

Studies done in ischemia models demonstrate that FTY720 decreases brain edema, reduces neuronal cell death, and improves long-term neurobehavioral outcome $[15,17,49]$ Furthermore, the early treatment of intracerebral hemorrhage patients with FTY720 reduces hematomaassociated edema and improves neurological outcome at 3 months [50]. Since SAH is associated with unique pathogenic mechanisms, the extrapolation of findings gathered in other stroke models should be done with caution. Current data, however, demonstrate that FTY720 has pleiotropic effects and can influence key elements of SAH, including neuronal survival, BBB permeability, neuroinflammation, and microvascular dysfunction $[15,17,49]$.

Similar to other brain-injury models, a profound immunodepression develops in SAH patients [51]. This temporary state lasts for at least 3 days after the bleed and is associated with a high rate of infections, particularly pneumonia. Thus, the potential translation of our findings to clinical practice may be limited by the immunomodulatory nature of fingolimod. Fingolimod is known to retain naïve $\mathrm{T}$ cells and central memory $\mathrm{T}$ cells in lymph nodes. However, this drug has a partial effect on peripheral effector memory $\mathrm{T}$ cells, which are important against infections [14]. There is limited information regarding the effect of fingolimod on infection rates on stroke. However, studies done in the rodent model of cerebral ischemia have shown that treatment with fingolimod reduces circulating leukocytes and improves outcome without increasing the rate of bacterial lung infections [52]. In addition, two recent small studies investigating the effect of fingolimod on patients with ischemic stroke and intracerebral hemorrhage showed similar rates of infection in both the treatment and placebo groups [50,53].

\section{Conclusion}

Early treatment with fingolimod after SAH reduces leukocyte adhesion to pial venules, preserves arteriolar dilation function, and improves neurological outcome. Further studies are needed to determine the long-term effect of fingolimod on SAH outcome.

\section{Abbreviations}

Ach: Acetylcholine; ADO: Adenosine; ANOVA: Analysis of variance; BBB: Blood brain barrier; CCA: Common carotid artery; CSF: Cerebrospinal fluid; ECA: External carotid arteries; FDA: Food and drug administration; FTY720: Fingolimod; ICA: Internal carotid artery; ICP: Intracranial pressure; IP: Intraperitoneal; LDF: Laser doppler flowmetry; MCA: Middle cerebral artery; NO: Nitric oxide; PTFE: Polytetrafluoroethylene; R6G: Rhodamine-6G; rCBF: Regional cerebral blood flow; S1P: Sphingosine-1-phosphate; SAH: Subarachnoid hemorrhage; SNAP: S-nitroso-N-acetyl penicillamine; VAP-1: Vascular adhesion protein-1.

\section{Competing interests}

The authors declare that they have no competing interests.

\section{Authors' contributions}

HLX and FDT were involved in the design of the study, carried out the experiments, and participated in the data analysis and manuscript preparation. DAP and CP participated in the data analysis and manuscript preparation. All authors read and approved the final manuscript.

\section{Ackowledgements}

Funded by minority recruitment supplement at UIC to FDT and R01-NS063279-04 from the NIH to DAP. 


\section{Author details}

${ }^{1}$ Neuroanesthesia Research Laboratory, University of Illinois College of Medicine, Chicago, IL, USA. ${ }^{2}$ Department of Anesthesiology of the University of Illinois College of Medicine, Chicago, IL, USA. ${ }^{3}$ Department of Neurology and Rehabilitation of the University of Illinois College of Medicine, $912 \mathrm{~S}$ Wood Street, Rm 855 N NPI (MC 796), Chicago, IL 60612-7330, USA.

\section{Received: 21 August 2014 Accepted: 1 January 2015} Published online: 27 January 2015

\section{References}

1. Huang J, van Gelder JM. The probability of sudden death from rupture of intracranial aneurysms: a meta-analysis. Neurosurgery. 2002;51:1101-5. discussion 1105-7.

2. Hop JW, Rinkel GJ, Algra A, van Gijn J. Case-fatality rates and functional outcome after subarachnoid hemorrhage: a systematic review. Stroke. 1997;28:660-4.

3. Mayer SA, Kreiter KT, Copeland D, Bernardini GL, Bates JE, Peery S, et al. Global and domain-specific cognitive impairment and outcome after subarachnoid hemorrhage. Neurology. 2002;59:1750-8.

4. Suarez Jl, Tarr RW, Selman WR. Aneurysmal subarachnoid hemorrhage. N Engl J Med. 2006;354:387-96.

5. Johnston SC, Mendis S, Mathers CD. Global variation in stroke burden and mortality: estimates from monitoring, surveillance, and modelling. Lancet Neurol. 2009;8:345-54.

6. Macdonald RL, Jaja B, Cusimano MD, Etminan N, Hanggi D, Hasan D, et al. SAHIT Investigators - on the outcome of some subarachnoid hemorrhage clinical trials. Transl Stroke Res. 2013;4:286-96.

7. Macdonald RL. Delayed neurological deterioration after subarachnoid haemorrhage. Nat Rev Neurol. 2014;10:44-58.

8. Schneider UC, Schiffler J, Hakiy N, Horn P, Vajkoczy P. Functional analysis of Pro-inflammatory properties within the cerebrospinal fluid after subarachnoid hemorrhage in vivo and in vitro. J Neuroinflammation. 2012;9:28. 2094-9-28.

9. Kooijman E, Nijboer $\mathrm{CH}$, van Velthoven CT, Mol W, Dijkhuizen RM, Kesecioglu J, et al. Long-term functional consequences and ongoing cerebral inflammation after subarachnoid hemorrhage in the rat. PLoS One. 2014;9:e90584.

10. Tobin MK, Bonds JA, Minshall RD, Pelligrino DA, Testai FD, Lazarov O. Neurogenesis and inflammation after ischemic stroke: what is known and where we go from here. J Cereb Blood Flow Metab. 2014;34:1573-84.

11. Fassbender K, Hodapp B, Rossol S, Bertsch T, Schmeck J, Schutt S, et al Inflammatory cytokines in subarachnoid haemorrhage: association with abnormal blood flow velocities in basal cerebral arteries. J Neurol Neurosurg Psychiatry. 2001;70:534-7

12. Lad SP, Hegen H, Gupta G, Deisenhammer F, Steinberg GK. Proteomic biomarker discovery in cerebrospinal fluid for cerebral vasospasm following subarachnoid hemorrhage. J Stroke Cerebrovasc Dis. 2012;21:30-41.

13. Soliven B, Miron V, Chun J. The neurobiology of sphingosine 1-phosphate signaling and sphingosine 1-phosphate receptor modulators. Neurology. 2011;76:S9-14.

14. Brinkmann V, Billich A, Baumruker T, Heining P, Schmouder R, Francis G, et al. Fingolimod (FTY720): discovery and development of an oral drug to treat multiple sclerosis. Nat Rev Drug Discov. 2010;9:883-97.

15. Wei Y, Yemisci M, Kim HH, Yung LM, Shin HK, Hwang SK, et al. Fingolimod provides long-term protection in rodent models of cerebral ischemia. Ann Neurol. 2011;69:119-29.

16. Rolland 2nd WB, Manaenko A, Lekic T, Hasegawa Y, Ostrowski R, Tang J, et al. FTY720 is neuroprotective and improves functional outcomes after intracerebral hemorrhage in mice. Acta Neurochir Suppl. 2011;111:213-7.

17. Hasegawa $Y$, Suzuki H, Sozen T, Rolland W, Zhang JH. Activation of sphingosine 1-phosphate receptor-1 by FTY720 is neuroprotective after ischemic stroke in rats. Stroke. 2010;41:368-74.

18. Sugawara $T$, Ayer $\mathrm{R}$, Jadhav $\mathrm{V}$, Zhang $\mathrm{JH}$. A new grading system evaluating bleeding scale in filament perforation subarachnoid hemorrhage rat model. J Neurosci Methods. 2008;167:327-34.

19. Xu HL, Paisansathan C, Pelligrino DA. Closed cranial window applications in the rat: studies on neurovascular coupling involving pial arterioles and the glia limitans. In: Zhao M et al., editors. Neurovascular coupling methods. New York: Springer Science+Business Media; 2014. p. 179-200.

20. $\mathrm{Xu} \mathrm{HL}$, Baughman VL, Pelligrino DA. Estrogen replacement treatment in diabetic ovariectomized female rats potentiates postischemic leukocyte adhesion in cerebral venules. Stroke. 2004;35:1974-8.
21. Xu HL, Galea E, Santizo RA, Baughman VL, Pelligrino DA. The key role of caveolin-1 in estrogen-mediated regulation of endothelial nitric oxide synthase function in cerebral arterioles in vivo. J Cereb Blood Flow Metab. 2001;21:907-13.

22. Britz GW, Meno JR, Park IS, Abel TJ, Chowdhary A, Nguyen TS, et al. Timedependent alterations in functional and pharmacological arteriolar reactivity after subarachnoid hemorrhage. Stroke. 2007;38:1329-35.

23. Rolland WB, Lekic T, Krafft PR, Hasegawa Y, Altay O, Hartman R, et al. Fingolimod reduces cerebral lymphocyte infiltration in experimental models of rodent intracerebral hemorrhage. Exp Neurol. 2013;241:45-55.

24. Hasegawa Y, Suzuki H, Altay O, Rolland W, Zhang JH. Role of the sphingosine metabolism pathway on neurons against experimental cerebral ischemia in rats. Transl Stroke Res. 2013:4:524-32.

25. Chun J, Hartung HP. Mechanism of action of oral fingolimod (FTY720) in multiple sclerosis. Clin Neuropharmacol. 2010:33:91-101.

26. Liesz A, Sun L, Zhou W, Schwarting S, Mracsko E, Zorn M, et al. FTY720 reduces post-ischemic brain lymphocyte influx but does not improve outcome in permanent murine cerebral ischemia. PLoS One. 2011;6:e21312.

27. Sabri M, Lass E, Macdonald RL. Early brain injury: a common mechanism in subarachnoid hemorrhage and global cerebral ischemia. Stroke Res Treat. 2013;2013:394036.

28. Chamorro A, Meisel A, Planas AM, Urra X, van de Beek D, Veltkamp R. The immunology of acute stroke. Nat Rev Neurol. 2012:8:401-10.

29. ladecola C, Anrather J. The immunology of stroke: from mechanisms to translation. Nat Med. 2011;17:796-808.

30. Kubota T, Handa Y, Tsuchida A, Kaneko M, Kobayashi H, Kubota T. The kinetics of lymphocyte subsets and macrophages in subarachnoid space after subarachnoid hemorrhage in rats. Stroke. 1993;24:1993-2000. discussion 2000-1.

31. Kooijman E, Nijboer CH, van Velthoven CT, Kavelaars A, Kesecioglu J, Heijnen CJ. The rodent endovascular puncture model of subarachnoid hemorrhage: mechanisms of brain damage and therapeutic strategies. J Neuroinflammation. 2014;11:2. 2094-11-2.

32. Kasius KM, Frijns CJ, Algra A, Rinkel GJ. Association of platelet and leukocyte counts with delayed cerebral ischemia in aneurysmal subarachnoid hemorrhage. Cerebrovasc Dis. 2010;29:576-83.

33. McMahon CJ, Hopkins S, Vail A, King AT, Smith D, Illingworth KJ, et al. Inflammation as a predictor for delayed cerebral ischemia after aneurysmal subarachnoid haemorrhage. J Neurointerv Surg. 2013;5:512-7.

34. Xu HL, Garcia M, Testai F, Vetri F, Barabanova A, Pelligrino DA, et al. Pharmacologic blockade of vascular adhesion protein-1 lessens neurologic dysfunction in rats subjected to subarachnoid hemorrhage. Brain Res. 2014;1586:83-9.

35. Mestas J, Hughes CC. Of mice and not men: differences between mouse and human immunology. J Immunol. 2004;172:2731-8.

36. Gomis P, Graftieaux JP, Sercombe R, Hettler D, Scherpereel B, Rousseaux P. Randomized, double-blind, placebo-controlled, pilot trial of high-dose methylprednisolone in aneurysmal subarachnoid hemorrhage. J Neurosurg. 2010;112:681-8.

37. Chyatte D, Fode NC, Nichols DA, Sundt Jr TM. Preliminary report: effects of high dose methylprednisolone on delayed cerebral ischemia in patients at high risk for vasospasm after aneurysmal subarachnoid hemorrhage. Neurosurgery. 1987;21:157-60.

38. Chyatte D, Sundt Jr TM. Response of chronic experimental cerebral vasospasm to methylprednisolone and dexamethasone. J Neurosurg. 1984;60:923-6.

39. Pradilla G, Chaichana KL, Hoang S, Huang J, Tamargo RJ. Inflammation and cerebral vasospasm after subarachnoid hemorrhage. Neurosurg Clin N Am. 2010:21:365-79.

40. Chaichana KL, Pradilla G, Huang J, Tamargo RJ. Role of inflammation (leukocyte-endothelial cell interactions) in vasospasm after subarachnoid hemorrhage. World Neurosurg. 2010;73:22-41.

41. Pradilla G, Wang PP, Legnani FG, Ogata L, Dietsch GN, Tamargo RJ Prevention of vasospasm by anti-CD11/CD18 monoclonal antibody therapy following subarachnoid hemorrhage in rabbits. J Neurosurg. 2004;101:88-92.

42. Friedrich B, Muller F, Feiler S, Scholler K, Plesnila N. Experimental subarachnoid hemorrhage causes early and long-lasting microarterial constriction and microthrombosis: an in-vivo microscopy study. J Cereb Blood Flow Metab. 2012;32:447-55.

43. Sun BL, Zheng CB, Yang MF, Yuan H, Zhang SM, Wang LX. Dynamic alterations of cerebral pial microcirculation during experimental subarachnoid hemorrhage. Cell Mol Neurobiol. 2009;29:235-41. 
44. Ostergaard L, Aamand R, Karabegovic S, Tietze A, Blicher JU, Mikkelsen IK, et al. The role of the microcirculation in delayed cerebral ischemia and chronic degenerative changes after subarachnoid hemorrhage. J Cereb Blood Flow Metab. 2013:33:1825-37.

45. Tolle M, Levkau B, Keul P, Brinkmann V, Giebing G, Schonfelder G, et al. Immunomodulator FTY720 induces eNOS-dependent arterial vasodilatation via the lysophospholipid receptor S1P3. Circ Res. 2005;96:913-20.

46. Hait NC, Wise LE, Allegood JC, O'Brien M, Avni D, Reeves TM, et al. Active, phosphorylated fingolimod inhibits histone deacetylases and facilitates fear extinction memory. Nat Neurosci. 2014;17:971-80.

47. Brunkhorst R, Vutukuri R, Pfeilschifter W. Fingolimod for the treatment of neurological diseases-state of play and future perspectives. Front Cell Neurosci. 2014;8:283

48. Xiong Y, Hla T. S1P control of endothelial integrity. Curr Top Microbiol Immunol. 2014;378:85-105.

49. Campos F, Qin T, Castillo J, Seo JH, Arai K, Lo EH, et al. Fingolimod reduces hemorrhagic transformation associated with delayed tissue plasminogen activator treatment in a mouse thromboembolic model. Stroke. 2013:44:505-11.

50. Fu Y, Hao J, Zhang N, Ren L, Sun N, Li YJ, et al. Fingolimod for the treatment of intracerebral hemorrhage: A 2-arm proof-of-concept study. JAMA Neurol. 2014;71:1092-101.

51. Sarrafzadeh A, Schlenk F, Meisel A, Dreier J, Vajkoczy P, Meisel C. Immunodepression after aneurysmal subarachnoid hemorrhage. Stroke. 2011;42:53-8.

52. Pfeilschifter W, Czech-Zechmeister B, Sujak M, Foerch C, Wichelhaus TA, Pfeilschifter J. Treatment with the immunomodulator FTY720 does not promote spontaneous bacterial infections after experimental stroke in mice. Exp Transl Stroke Med. 2011;3:2. 7378-3-2.

53. Fu Y, Zhang N, Ren L, Yan Y, Sun N, Li YJ, et al. Impact of an immune modulator fingolimod on acute ischemic stroke. Proc Natl Acad Sci U S A. 2014;111:18315-20.

\section{Submit your next manuscript to BioMed Central and take full advantage of:}

- Convenient online submission

- Thorough peer review

- No space constraints or color figure charges

- Immediate publication on acceptance

- Inclusion in PubMed, CAS, Scopus and Google Scholar

- Research which is freely available for redistribution 